\title{
Efecto de la aplicación de cachaza fresca y de dos sistemas de producción maíz y maíz con asocio fríjol sobre las propiedades físicas de un Inceptisol
}

\section{Effect of application of fresh filter cake and two production systems of corn and corn associated with beans on the physical properties of an Inceptisol}
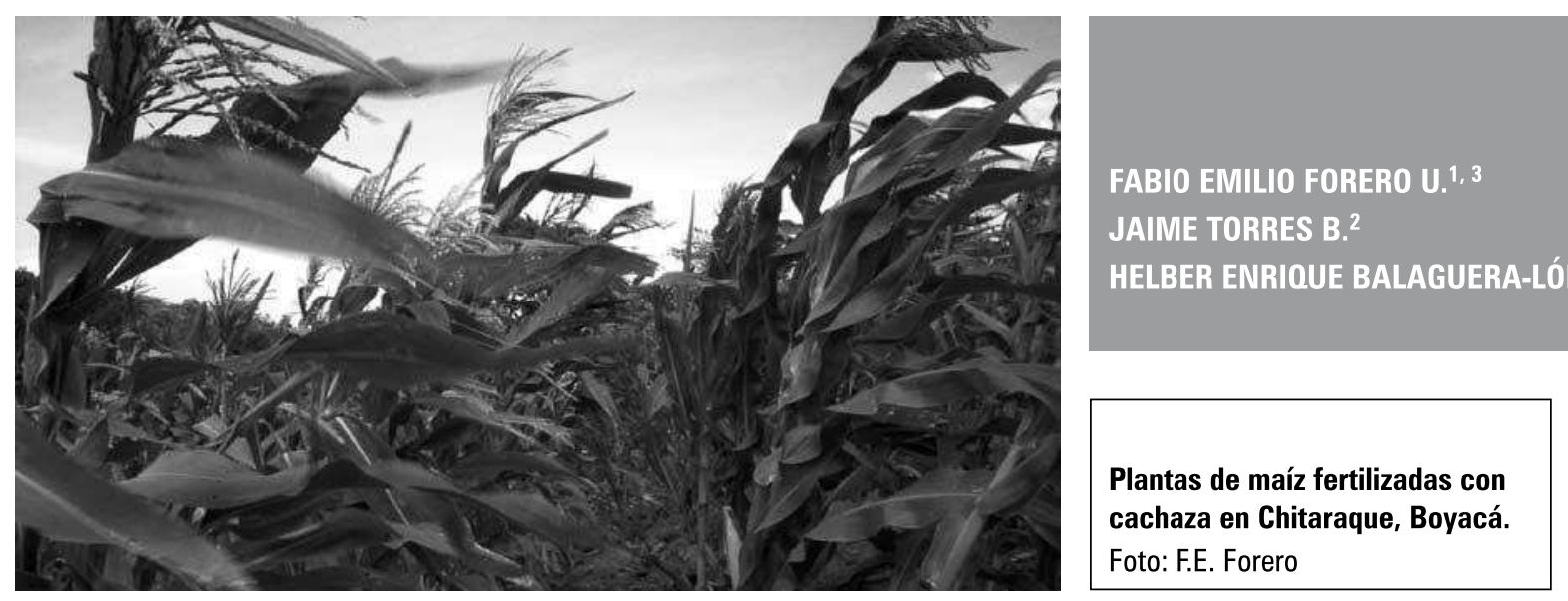

\section{Resumen}

La cachaza fresca de trapiche panelero es un subproducto obtenido del proceso de elaboración de panela, que tiene alto potencial como enmienda orgánica. Sin embargo, este residuo que equivale al $3 \%$ de la caña molida es arrojado a las fuentes hídricas o cerca de los trapiches generando problemas graves de contaminación ambiental. En la zona de la Hoya del Río Suárez (Boyacá, Colombia) el principal cultivo es la caña panelera, pero es apta para cultivos de pancoger como el maíz y el fríjol. Con el objetivo de evaluar el efecto de la cachaza fresca de trapiche panelero y dos sistemas de producción (maíz y maíz asocio fríjol) sobre las propiedades físicas de un Inceptisol, se aplicaron cuatro dosis de cachaza (0, 5, 10 ó $\left.15 \mathrm{t} \mathrm{ha}^{-1}\right)$ a cada uno de los sistemas de producción. Después de la cosecha se encontró que el sistema maíz asocio fríjol presentó el mayor diámetro ponderado medio en seco y húmedo y mayor porosidad, estas propiedades físicas junto con la densidad aparente fueron favorecidas con el incremento de la aplicación de cachaza. Con la aplicación de $15 \mathrm{t} \mathrm{ha} \mathrm{a}^{-1} \mathrm{de}$ cachaza en el sistema maíz asocio fríjol se garantizó mayor efecto sobre las propiedades físicas del suelo.

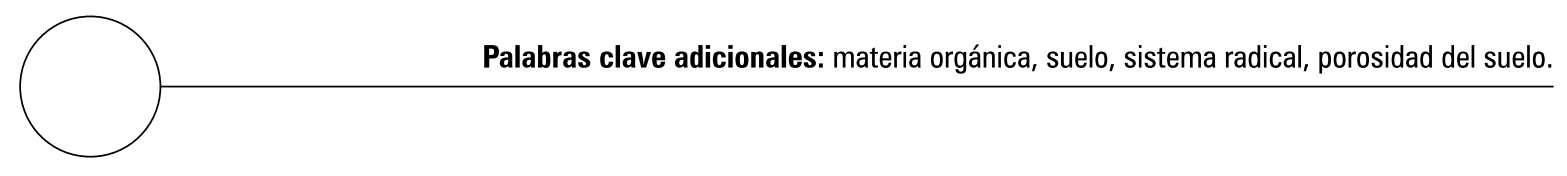

\footnotetext{
Grupo de Investigaciones Agrícolas, Facultad de Ciencias Agropecuarias, Universidad Pedagógica y Tecnológica de Colombia, Tunja (Colombia).

2 Departamento de Agronomía, Facultad de Agronomía, Universidad Nacional de Colombia, Bogotá (Colombia).

3 Autor para correspondencia.guatoquero@gmail.com
} 


\section{Abstract}

The fresh filter cake of sugar cane mill is a byproduct of the process from the manufacturing of brown sugarloaf which has high potential as organic amendment. However, this waste is equivalent to $3 \%$ cane crushed that is discharged to water sources or thrown near the sugar mills creating serious problems of environmental contamination. In the "Hoya del Rio Suarez" zone (Boyaca, Colombia), the main crop is the sugar cane, but this land is suitable for other crops, such as corn and bean. In order to evaluate the effect of fresh filter cake of sugar cane mill and that of two production systems (maize and maize associated with bean) on the physical properties of the Inceptisol, four doses of filter cake $\left(0,5,10\right.$ or $\left.15 \mathrm{t} \mathrm{ha}^{-1}\right)$ were applied to each of the production systems. After the harvest it was found that corn associated with beans had the greatest average weighted diameter dried and humid and the porosity; these properties together with bulk density were favored with the increase in the application of filter cake. With the application of $15 \mathrm{tha}^{-1}$ the filter cake in the system maize associated with bean guaranteed the major effect on soil physical properties.

Additional key words: organic matter, soil, root system, soil porosity.

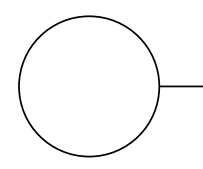

La cachaza es un material marrón oscuro, constituido por una mezcla de fibra de caña, sacarosa, coloides, coagulados, incluyendo la cera, fosfato de calcio y partículas de suelo (Zurro, 2005). Es considerada como el subproducto más importante de los ingenios azucareros y en la agroindustria artesanal de la caña panelera en la producción de panela, con cierto potencial como fertilizante y mejorador de las propiedades físicas del suelo (Zérega, 1993), producida a una tasa de 3 t húmedas por cada 100 t de caña molida. La cachaza tiene alto contenido de materia orgánica y presenta potencial como acondicionador de suelos, ya que aporta cantidades altas de humus necesarias para mantener la fertilidad de suelos en zonas tropicales y subtropicales (Mohee y Panray, 1999).

En el año 2006, de la cachaza producida en la región de la Hoya del Río Suárez y Cundinamarca, se estaba utilizando $47 \%$ como alimento para animales domésticos y el 53\% restante que corresponde aproximadamente a $21.812 \mathrm{t}$ año-1 se abandonaba al aire libre (Rodríguez y Gottret, 2006), incluyendo los cursos de agua, causando graves problemas de contaminación ambiental, ya que presenta una elevada demanda bioquímica de oxígeno, genera malos olores y atrae insectos, roedores y otras plagas.

En la región de la Hoya del Río Suárez, se desconoce el uso que puede tener la cachaza en la producción agrícola como aporte de materia orgánica (Arrieche y Mora, 2005), masa microbial (Khan et al., 2008) y nutrientes (Paturau, 1969), también se ha encontrado que afecta la salinidad (Rasul et al., 2006), corrige acidez del suelo (Zérega, 1993), y principalmente, presenta alto potencial como mejorador de las propiedades físicas del suelo (Painuli y Abrol, 1986).

Además, la costumbre ancestral es la siembra del monocultivo de la caña panelera, sin tener en cuenta que existen otros cultivos como el maíz (Zea mayz L.) y maíz en asocio con especies como el fríjol (Phaseolus vulgaris L.), los cuales son una 
importante fuente de ingresos para muchos productores, y pueden ser una alternativa adecuada para la rotación de cultivos que permitan mantener o mejorar las propiedades físicas de los suelos de la zona.

Por lo anterior, el objetivo de esta investigación fue evaluar el efecto de la aplicación de cachaza fresca de trapiche panelero y de dos sistemas de producción maíz y maíz asocio fríjol sobre las propiedades físicas de un Inceptisol presente en la zona panelera de la Hoya del Río Suárez, con el propósito de darle una alternativa de uso a la cachaza producida en la zona.

\section{MATERIALES Y MÉTODOS}

El trabajo de campo fue desarrollado en la Finca La Florida del municipio de Chitaraque (Boyacá), que se encuentra ubicada a 5059'87" N y 7327'78" W, con una altura de $1.729 \mathrm{msnm}$. Las condiciones climáticas de la zona durante el periodo del experimento (4 meses) fueron las siguientes: precipitación $660 \mathrm{~mm}$, temperatura promedio de $18,6^{\circ} \mathrm{C}$, temperatura máxima promedio $25,9^{\circ} \mathrm{C}$, temperatura mínima promedio $13^{\circ} \mathrm{C}$ y humedad relativa promedio del $80 \%$. Los análisis físicos de suelos se realizaron en el laboratorio de docencia de suelos de la Facultad de Ciencias Agropecuarias de la Universidad Pedagógica y Tecnológica de Colombia, Tunja.

Se utilizó un diseño experimental en bloques completamente al azar, donde los bloques correspondieron a dos sistemas de producción (maíz y maíz asocio fríjol), cada bloque fue repetido tres veces, dentro de cada bloque se distribuyeron al azar cuatro dosis de cachaza fresca de trapiche panelero $\left(0,5,10\right.$ ó $\left.15 \mathrm{t} \mathrm{ha}^{-1}\right)$, en total fueron 24 unidades experimentales (UE). Cada UE correspondió a una parcela de $6 \times 4 \mathrm{~m}$, separadas entre si a $0,5 \mathrm{~m}$.

Para el sistema maíz se sembraron tres semillas de maíz por sitio con distancias de siembra de
0,9 m x 0,45 m. Después de la emergencia se dejaron solamente dos plántulas por sitio. En el sistema asocio maíz fríjol se utilizaron las mismas distancias de siembra para el maíz, y 15 días después de la siembra del maíz, en cada sitio se sembraron dos semillas de fríjol para finalmente dejar una planta. Se utilizó maíz ICA V-305 y fríjol cargamanto rojo.

La preparación del suelo se realizó de acuerdo a lo sugerido por los agricultores de la zona, mediante arado con bueyes y dos cruzas. Luego, se realizó el trazo según el diseño experimental y se procedió a aplicar la cachaza fresca de trapiche panelero, la cual fue aplicada manualmente al voleo y se incorporó con azadón, la siembra se realizó 20 d después de esta actividad, pues es necesario que la cachaza reaccione en el suelo y pierda su actividad fitotóxica, generada por el alto contenido de $\mathrm{CO}_{2}$ y $\mathrm{CH}_{4}$ que este material orgánico presenta (Zérega, 1993). La composición química de la cachaza fresca de trapiche panelero se presenta en la tabla 2.

Tabla 1. Propiedades fisicoquímicas del suelo antes de la siembra.

\begin{tabular}{|l|r|l|}
\hline \multicolumn{1}{|c|}{ Propiedad } & Valor & \multicolumn{1}{c|}{ Unidades } \\
\hline Arena & 42,88 & $\%$ \\
\hline Arcilla & 29,12 & $\%$ \\
\hline Limo & 28,00 & $\%$ \\
\hline $\mathrm{pH}$ & 4,60 & \\
\hline Carbono orgánico & 4,12 & $\%$ \\
\hline $\mathrm{Ca}$ & 3,41 & $\mathrm{cmol} \mathrm{kg}$ \\
\hline $\mathrm{Mg}$ & 0,56 & $\mathrm{cmol} \mathrm{kg}^{-1}$ de suelo \\
\hline $\mathrm{K}$ & 0,41 & $\mathrm{cmol} \mathrm{kg}^{-1}$ de suelo \\
\hline $\mathrm{Na}$ & 0,46 & $\mathrm{cmol} \mathrm{kg}^{-1}$ de suelo \\
\hline $\mathrm{P}$ & 8,50 & $\mathrm{mg} \mathrm{kg}^{-1}$ de suelo \\
\hline $\mathrm{Fe}$ & 97,70 & $\mathrm{mg} \mathrm{kg}^{-1}$ de suelo \\
\hline $\mathrm{Cu}$ & 2,43 & $\mathrm{mg} \mathrm{kg}^{-1}$ de suelo \\
\hline $\mathrm{Zn}$ & 3,00 & $\mathrm{mg} \mathrm{kg}^{-1}$ de suelo \\
\hline $\mathrm{Mn}$ & 1,93 & $\mathrm{mg} \mathrm{kg}^{-1}$ de suelo \\
\hline $\mathrm{Al}+3$ & 5,10 & $\mathrm{cmol} \mathrm{kg}^{-1}$ de suelo \\
\hline $\mathrm{H}^{+}$ & 0,10 & $\mathrm{cmol} \mathrm{kg}$ \\
\hline $\mathrm{CICe}$ & 10,04 & $\mathrm{cmol} \mathrm{kg}$ de suelo \\
\hline $\mathrm{CE}$ & 0,70 & $\mathrm{dS} \mathrm{m}{ }^{-1}$ \\
\hline
\end{tabular}

Taxonomía: Typic Dystrudepts. 
Se realizó un muestreo de suelos previo a la preparación del mismo, con el fin de determinar las condiciones iniciales del experimento, para lo cual, se tomaron en zigzag seis submuestras a $20 \mathrm{~cm}$ de profundidad en toda el área experimental con barreno holandés, las submuestras se mezclaron y se obtuvo una muestra de $1 \mathrm{~kg}$ para los análisis fisicoquímicos, cuyos resultados se reportan en la tabla 1. Este procedimiento fue repetido después de la cosecha (4 meses después de la siembra), no obstante, el muestreo se realizó para cada UE y se cuantificó el diámetro ponderado medio seco (dpms) y húmedo (dpmh) siguiendo la metodología propuesta por el IGAC (2006). Además, se determinó la densidad aparente con el muestreador Bulman (0200, Soil Care Sampler), densidad real mediante método del picnómetro y porosidad total del suelo.

\section{Tabla 2. Composición química de la cachaza fresca} de trapiche panelero.

\begin{tabular}{|l|c|}
\hline \multicolumn{1}{|c|}{ Atributo } & Valor \\
\hline$\%$ ceniza & 8,9 \\
\hline$\%$ de humedad & 49,625 \\
\hline$\%$ de materia seca & 50,375 \\
\hline$\%$ de MO & 21,1 \\
\hline$\% \mathrm{CO}$ & 12,23 \\
\hline$\% \mathrm{~N}$ & 1,15 \\
\hline$\% \mathrm{Ca}$ & 6,52 \\
\hline$\% \mathrm{Mg}$ & 0,66 \\
\hline$\% \mathrm{~K}$ & 0,735 \\
\hline$\% \mathrm{Na}$ & 0,3 \\
\hline$\% \mathrm{P}$ & 0,074 \\
\hline$\% \mathrm{Cu}$ & 0,05 \\
\hline$\% \mathrm{Zn}$ & 0,0755 \\
\hline$\% \mathrm{Mn}$ & 0,057 \\
\hline$\% \mathrm{Fe}$ & 0,0465 \\
\hline $\mathrm{CE}$ & 3,7 \\
\hline $\mathrm{pH}$ & 4,0 \\
\hline $\mathrm{C} / \mathrm{N}$ & 10,445 \\
\hline
\end{tabular}

Los datos obtenidos fueron sometidos a un análisis de varianza (Anava) y se les realizó la prueba de comparación múltiple de Tukey con una confiabilidad del 95\%. Para el análisis de los datos se utilizó el paquete estadístico SAS v.8e (Cary, NC.).

\section{RESULTADOS Y DISCUSIÓN}

El dpms fue afectado significativamente por el sistema de cultivo $(P \leq 0,05)$, el sistema asocio maíz fríjol se caracterizó por aumentar en mayor grado los agregados, en este mismo sistema se observó que el dpms se incrementó linealmente en función de la dosis de cachaza aplicada $(P \leq 0,05)$, mientras que en el sistema de maíz en monocultivo no se presentaron diferencias estadísticas, no obstante, hubo mayor respuesta con $10 \mathrm{t} \mathrm{ha}^{-1}$ (figura 1).

El sistema asocio maíz frijol fue significativamente $(P \leq 0,05)$ más efectivo que el sistema maíz al generar mayor aumento del dpmh. Entre dosis de cachaza no hubo diferencias estadísticas, pero en los dos sistemas de cultivo se observó mayor respuesta con la dosis de $15 \mathrm{t} \mathrm{ha}^{-1}$ cachaza fresca de trapiche panelero (figura 2).

El sistema radical de las plantas de maíz y fríjol cumplen una función muy importante en la formación de agregados, pues, la influencia de la raíz puede ser mecánica, por el acomodo radicular que permite la unión temporal de partículas y fragmentos minerales, y también bioquímica debido a la producción de mucílagos, humus, gomas y otros compuestos orgánicos que actúan como enlaces transitorios y permanentes entre la fracción mineral, o bien, como cubiertas que protegen a los agregados incrementando su estabilidad (Velázquez-Rodríguez et al., 2001). No obstante, el aumento de la agregación del suelo puede ser optimizado con el aporte de materia orgánica de la cachaza y las raíces y a la solubilización de cementantes químicos. 


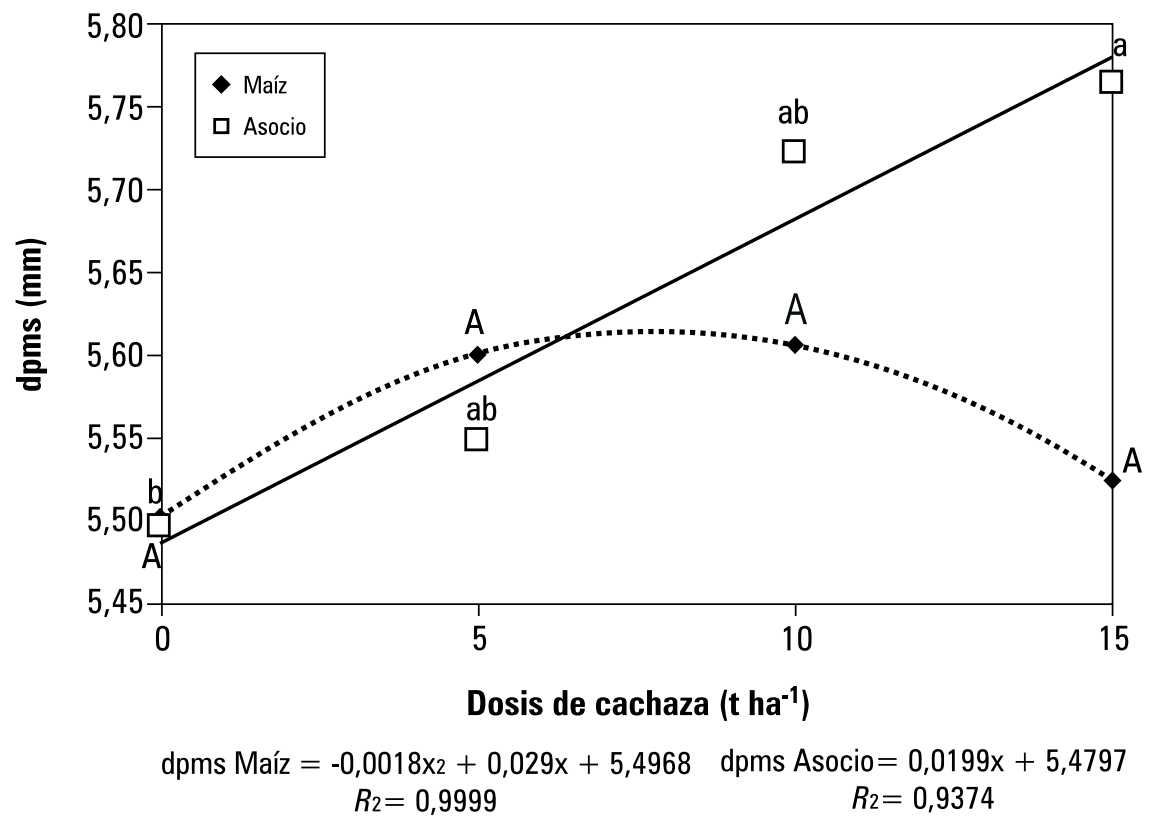

Figura 1. Efecto de la aplicación de cachaza fresca de trapiche panelero y de dos sistemas de cultivo sobre el diámetro ponderado medio seco (dpms) de un Inceptisol. Promedios con letras distintas en la misma serie indican diferencia significativa según la prueba de Tukey $(P \leq 0,05)$.

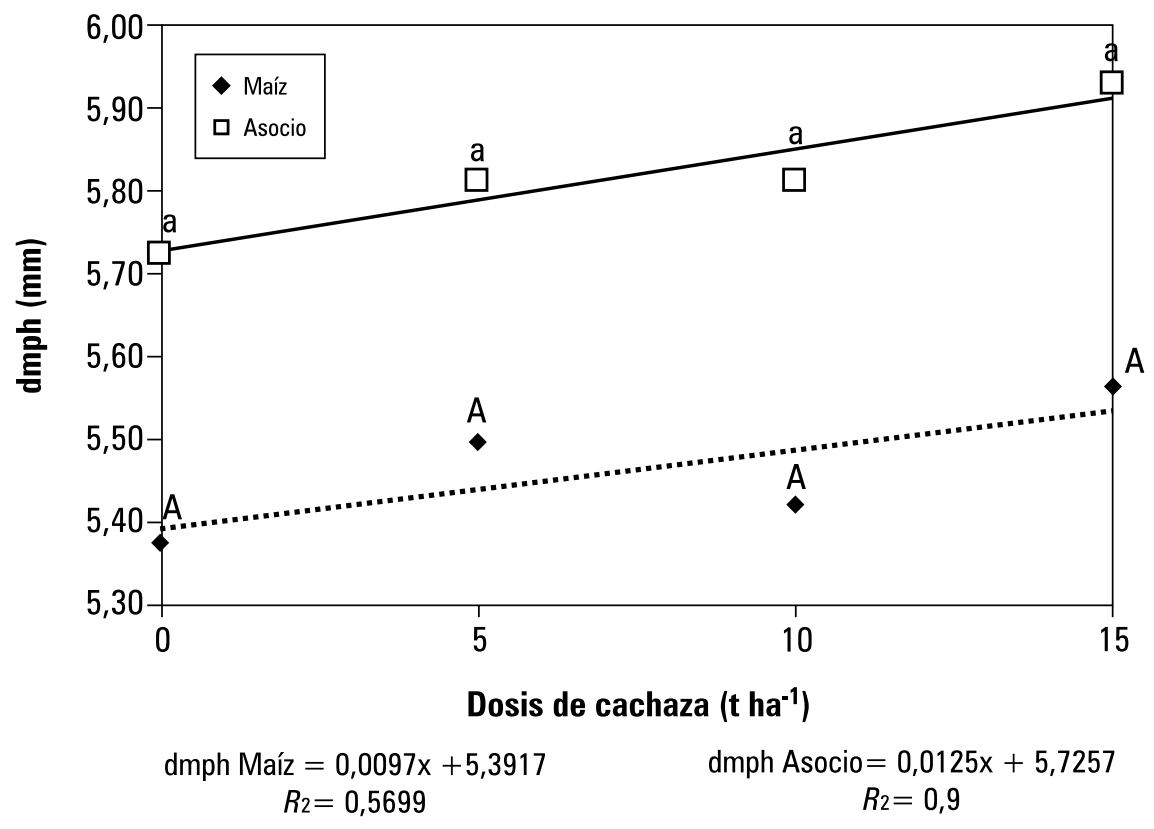

Figura 2. Efecto de la aplicación de cachaza fresca de trapiche panelero y de dos sistemas de cultivo sobre el diámetro ponderado medio húmedo (dpmh) de un Inceptisol. Promedios con letras distintas en la misma serie indican diferencia significativa según la prueba de Tukey $(P \leq 0,05)$. 
Al respecto, Carpenedo y Mielniczuk (1990) afirman que el tipo de vegetación interfiere en la estructura del suelo, por ejemplo, las gramíneas son más eficientes en aumentar y mantener la estabilidad de agregados que las leguminosas, por presentar un sistema radical más extenso y renovado constantemente (Harris et al., 1966). Sin embargo, el resultado que se encontró en el presente estudio indicó que el asocio maíz fríjol es más eficiente que la siembra de solo maíz, porque genera mayor densidad radicular y aporte de nitrógeno que aumentan la tasa de descomposición de los residuos como consecuencia de la disminución de la relación C/N (Carpenedo y Mielniczuk, 1990).

El efecto de la cachaza fresca de trapiche panelero como material orgánico junto con la materia orgánica que generan las raíces muertas de las plantas de maíz y frijol, en la formación de agregados corresponde a lo afirmado por Tisdall y Oades (1982), quienes demostraron que la materia orgánica ejerce un papel importante en la formación y estabilización de agregados del suelo debido a puentes entre polímeros orgánicos y una superficie orgánica mediada por cationes polivalentes. La materia orgánica actúa como agregante físico del suelo mediante su estructura compleja de largas cadenas de carbono que agregan las partículas minerales, además se encarga de proteger las partículas minerales de la acción del agua para evitar su disgregación y permitir así agregados más estables y persistentes en el suelo (Tisdall y Oades, 1982).

Cunha et al. (2007) determinaron que la materia orgánica es el principal agente agregante del suelo, ya que a medida que aumentó el aporte de materia seca de pasturas como material orgánico, también lo hizo el diámetro medio ponderado. Sánchez-Hernández et al. (2006) encontraron que el incremento en la dosis de vermicompost de cachaza aumentó la proporción de macroagregados estables en húmedo, específicamente los de tamaño mayor a $0,5 \mathrm{~mm}$. Los agregados menores a ese tamaño fueron más frecuentes en los suelos que recibieron menos cantidad de la enmienda. Solamente, los agregados mayores a 3,36 $\mathrm{mm}$ y entre $0,5-1,0 \mathrm{~mm}$ se diferenciaron estadísticamente, particularmente en los suelos que recibieron las dosis más altas.

Estos mismos autores encontraron que el dpms aumentó en relación directa a las dosis de la enmienda orgánica. De esta forma, el tratamiento de $60 \mathrm{t}$ ha ${ }^{-1}$ presentó el mayor efecto, mientras que en el testigo la respuesta fue menor (Sánchez-Hernández et al., 2006), lo cual coincide con lo encontrado en este estudio mediante la adición de cachaza. Campos et al. (1995) afirman que los compuestos orgánicos y la acción microbiana favorecen la estabilidad de agregados por la alta correlación encontrada entre el contenido de carbono orgánico (CO) y la actividad microbiana con el diámetro medio geométrico de los agregados. Similar respuesta obtuvieron en dpmh. Esta situación puede también ser una posible explicación del incremento del tamaño del agregado en función de la dosis de cachaza suministrada al suelo.

También es posible que la cachaza panelera promueva la liberación de gran cantidad de $\mathrm{CO}_{2}$ en su transformación formando $\mathrm{H}_{2} \mathrm{CO}_{3}$ y disuelva cationes como lo reportan algunos autores (Van Rooyen y Webber, 1977; Zérega, 1993), y que sumado el efecto de los ácidos liberados por las raíces de maíz y fríjol coadyuden a la liberación de calcio, que también tiene alto efecto agregante (Fassbender, 1986). Así, de acuerdo con un modelo propuesto por Edwards y Bremer (1967), los cationes polivalentes, principalmente el calcio son cruciales para unir la fracción orgánica y los minerales de arcilla, sin esa unión, la fracción arcilla y la orgánica se dispersan porque las dos tiene cargas negativas permanentes. La predominancia del calcio en el complejo de cambio, además de promover la floculación adecuada de las arcillas contribuye con el aumento de la actividad biológica que favorece la formación de agregados (Fassbender, 1986). 
Por otro lado, la acción conjunta de la cachaza y el efecto de los sistemas radiculares del maíz y el fríjol parece ser que crean un ambiente propicio para aumentar las poblaciones de hongos y bacterias. Estos microorganismos son capaces de unir la fracción mineral del suelo en agregados estables, aunque cada uno tiene una eficiencia específica para cumplir con este propósito (Harris et al., 1966).

No existe un consenso generalizado acerca del mecanismo general involucrado en la estabilización de los agregados, ya que en ello confluye una serie de interacciones físicas, químicas y biológicas (Harris et al., 1966). El crecimiento y la liberación de exudados radicales de diferente tipo, el incremento de policationes en la rizósfera, la producción de agentes cementantes por parte de la raíz y microorganismos, la naturaleza de la planta y condiciones de manejo agronómi- co, actividad de lombrices y mesofauna, hongos filamentosos y actinomicetos, entre otros, son algunos de los factores más incidentes en la formación de agregados estables (Velázquez-Rodríguez et al., 2001), lo que explica un mayor aumento en la formación de agregados cuando se da el efecto conjunto del sistema radical del maíz y el fríjol y la adición de cachaza.

La densidad aparente del suelo no presentó diferencias significativas en el sistema asocio. En el sistema maíz, una función cuadrática describió el comportamiento de la adición de cachaza sobre la densidad aparente, donde la aplicación de 10 t ha ${ }^{-1}$ de cachaza presentó la menor densidad aparente $(P \leq 0,05)$. Entre sistemas de cultivo no se presentaron diferencias estadísticas y los valores promedio fueron $1,08 \mathrm{~g} \mathrm{~cm}^{-3}$ para maíz y $1,09 \mathrm{~g} \mathrm{~cm}^{-3}$ para el sistema asocio (figura 3).

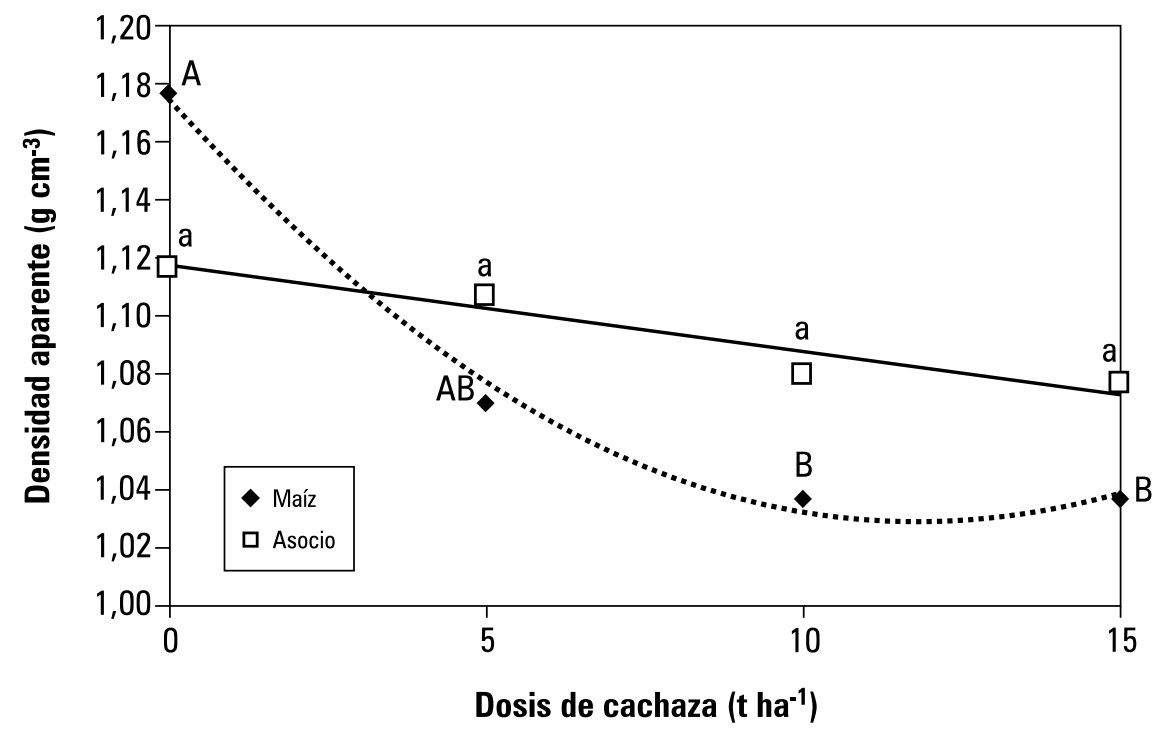

DA Asocio $=-0,0029 x+1,117$

$R_{2}=0,9219$
DA Maíz = 0,0011x2 -0,0251x + 1,1747

$R_{2}=0,9939$

Figura 3. Efecto de la aplicación de cachaza fresca de trapiche panelero y de dos sistemas de cultivo sobre la densidad aparente de un Inceptisol. Promedios con letras distintas en la misma serie indican diferencia significativa según la prueba de Tukey $(P \leq 0,05)$. 
Las especies vegetales contribuyen decisivamente en la recuperación de suelos, en la formación de agregados y en la modificación de los que tienen una estructura deficiente, lo que se relaciona directamente con el efecto del sistema radical en la fragmentación, reorganización y estabilización del material y aumento del volumen del suelo (Tisdall, 1994).

Con la adición de cachaza al suelo es posible mejorar la densidad aparente por su alto contenido de materia orgánica. Soane et al. (1981) determinaron que la materia orgánica tiene una correlación elevada y negativa con la densidad aparente, debido a que esta, por su menor densidad, produce dilución de la matriz del suelo y aumenta la estabilidad de agregados (MacRae y Mehuys, 1985). De esta manera, el agregado de materia orgánica aumenta la estabilidad estructural (Tisdall y Oades, 1982), disminuye la densidad aparente, mejora las tasas de infiltración de agua y la conductividad hidráulica (Felton et al., 1995).

La densidad aparente es una de las propiedades más determinantes de las características de permeabilidad y aireación del suelo, y su persistencia en el tiempo es reflejo de la estabilidad de los agregados. Así, diversos autores coinciden en señalar que aplicaciones de $\mathrm{MO}$ al suelo provocan una disminución de la densidad, como consecuencia de un aumento de la macroporosidad (Kay et al., 1997). Además, se obtienen incrementos notables en la infiltración del agua (Le Bissonnais y Arrouays, 1997) y un mayor contenido de agua aprovechable del suelo (Hudson, 1994). Por tanto, de la cachaza también se obtendrían efectos benéficos.

El aumento en la cantidad de raíces del sistema asocio incrementa la porosidad por acción mecánica, donde el crecimiento de raíces descompacta el suelo y aumenta su volumen, al igual que por el aporte de materia orgánica proveniente de raíces muertas, ya que según Hernández et al. (1995), la MO aportada por el sistema radical del cultivo de caña al suelo es de aproximadamente
$2,5 \mathrm{t} \mathrm{ha}^{-1}$ año-1, que se convierte en una cantidad considerable con acción favorable sobre la fertilidad del suelo. La menor densidad aparente es el resultado de la mayor macroporosidad, ya que se generan espacios dentro de la matriz del suelo por efecto del material orgánico adicionado. Mayores cantidades de materia orgánica adicionados generan menores valores de densidad aparente y aumentos en los valores de porosidad total.

Unger y Jones (1998) afirman que la adición de materia orgánica al suelo puede reducir la densidad aparente; no afectarla (Acosta et al., 1999), y en algunas situaciones aumentarla (Bottenberg et al., 1999). El efecto puede variar por el tipo de suelo, antecedentes de las propiedades del suelo, tipo de materia orgánica, clima, uso del suelo y cantidades aplicadas (Mulumba y Lal, 2008). Por tanto, para el caso de la adición de cachaza fresca es evidente que disminuye la densidad aparente, y su efecto es mayor al aumentar las dosis.

Por otro lado, Sánchez-Hernández et al. (2006) demostraron que el aporte de materiales orgánicos en suelos vertisoles durante dos años, disminuyó la densidad de 1,34 a $1,16 \mathrm{~g} \mathrm{~cm}^{-3}$, resultado que concuerda con lo reportado para cachaza en esta investigación.

Respecto a la porosidad total del suelo, no hubo diferencias estadísticas entre sistemas de cultivo, no obstante, el sistema asocio generó mayor porosidad $(57,1 \%)$ que el sistema maíz (55,81\%). En los dos sistemas de cultivo se aumentó la porosidad de forma lineal a medida que fue mayor el suministro de cachaza fresca $(P \leq 0,05)$ (figura 4).

La aireación es uno de los factores físicos más importantes que puede limitar el desarrollo de los sistemas radiculares de las plantas, el crecimiento y producción de los cultivos (Boone y Veen, 1994). La porosidad del suelo interfiere en la aireación, conducción y retención del agua, resistencia a la penetración y a la ramificación de raíces en el suelo, y por ende en el aprovechamiento de agua y nutrientes disponibles. Un aumento de la poro- 


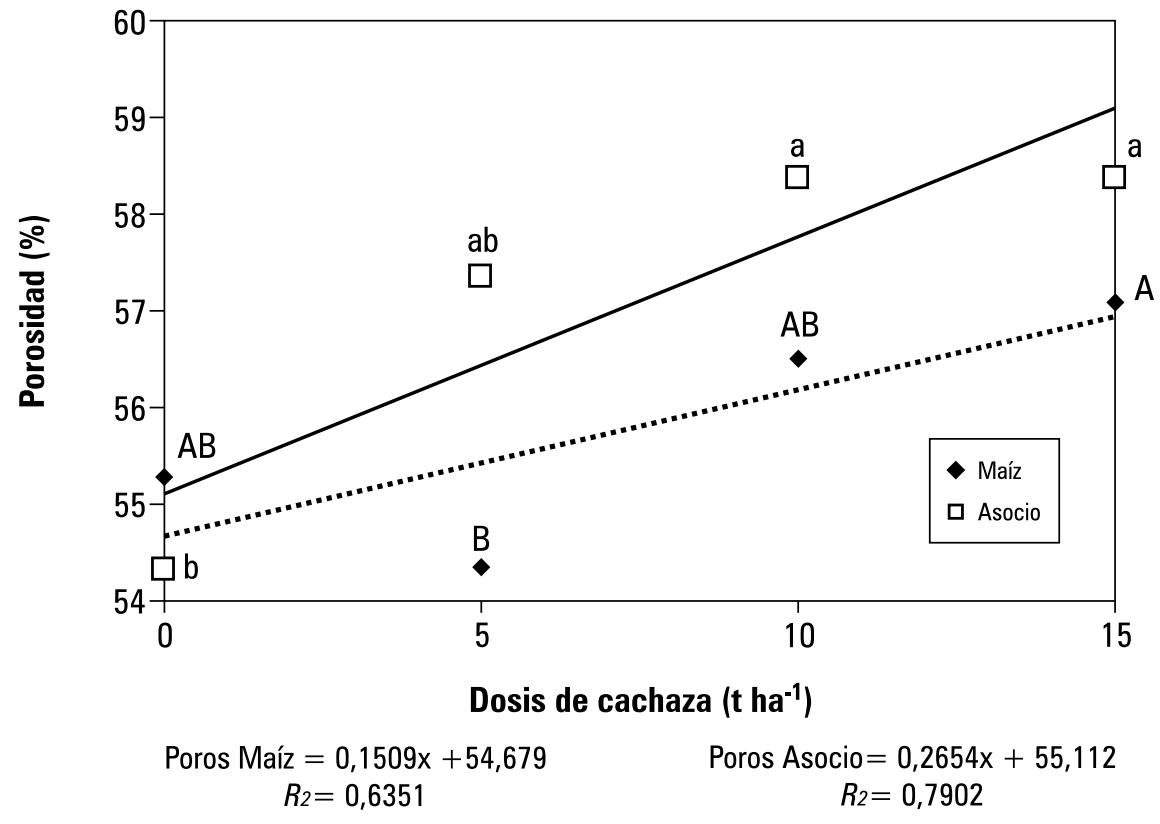

Figura 4. Efecto de la aplicación de cachaza fresca de trapiche panelero y de dos sistemas de cultivo sobre la porosidad total de un Inceptisol. Promedios con letras distintas en la misma serie indican diferencia significativa según la prueba de Tukey $(P \leq 0,05)$.

sidad es también el resultado de la descomposición de raíces y la liberación de exudados radiculares que generan una mayor actividad biológica del suelo, proporcionando mayor estabilidad de agregados (Tisdall y Oades, 1982).

Por lo anterior, las gramíneas incluyendo el maíz, por su sistema radical promueven un efecto positivo en la aireación del suelo y estabilidad de agregados (Silva y Mielniczuk, 1998), aunque es posible que el asocio con leguminosas contribuya a acelerar la descomposición de la materia orgánica, generando mayor aporte de nitrógeno y aumentando la porosidad del suelo (Cunha et al., 2007), lo constatado en la siembra de maíz asocio fríjol. Donde el mayor sistema radical logrado por el sistema asocio se reflejó en el mayor aumento en la porosidad del suelo estudiado. Lo que concuerda con el efecto del sistema radical de abonos verdes, que lograron la recuperación de un suelo degradado, mejorando la porosidad y revirtiendo la compactación subsuperficial (Píccolo, 1995).

El aumento de la porosidad del suelo observado en este experimento también puede ser debido al aporte de materia orgánica proveniente de la cachaza y a la descomposición de raíces de las plantas de maíz y fríjol, que son un aporte importante de materia orgánica y concuerda con Herrick y Lal (1995) quienes afirman que los aportes de materiales orgánicos disminuyen la densidad por mejoramiento en la estructura del suelo. Pues la variación de la porosidad depende del tamaño de las partículas y del grado de agregación, así, al favorecerse la formación de agregados hay un consecuente aumento de la porosidad. Además, la aplicación de materiales orgánicos mejora la 
estructura del suelo, incrementa la capacidad de aireación, lo que causa que las raíces se extiendan y exploren un mayor volumen de suelo, esto a su vez aumenta la retención de humedad a través del perfil de suelo (Pernes-Debuyser y Tessier, 2004).

Mulumba y Lal (2008) determinaron que el aporte de materia orgánica incrementó la porosidad siguiendo una curva descrita por un polinomio de segundo grado, donde la mayor porosidad se logró con 4 t ha ${ }^{-1}$ de materia orgánica. Emerson y McGarry (2003) encontraron que al aumentar el contenido de carbono orgánico, el volumen del suelo fue mayor debido a un aumento en el número de poros. Así, Cuevas et al. (2006) afirman que mayores cantidades de materia orgánica adicionadas generan menores valores de densidad aparente $y$ aumentos en los valores de porosidad total, favoreciendo el movimiento del aire y del agua.

Painuli y Abrol (1986) y Zérega (1993) recomiendan que para lograr efectos prolongados en la porosidad del suelo superiores a 3 años se debe hacer proporcionalmente mezclas de cachaza y enmiendas químicas como yeso o fosfoyeso; además, la mezcla de cachaza y materiales bajos en descomposición como bagazo por su alta relación $\mathrm{C} / \mathrm{N}$ son potencialmente importantes en el estudio para evaluar el mejoramiento de las propiedades físicas de los suelos.

\section{CONCLUSIONES}

Con la aplicación de 15 t ha ${ }^{-1}$ de cachaza fresca de trapiche panelero en el sistema maíz asocio fríjol, se logró un efecto favorable sobre las propiedades físicas del suelo, pues se aumentó el diámetro ponderado medio en seco y en húmedo y la porosidad, y se disminuyó la densidad aparente, convirtiéndose en una alternativa de manejo para los suelos de la zona cañera de la Hoya del Río Suárez, y también es una forma de darle un uso adecuado a la cachaza fresca y de esta manera disminuir la contaminación ambiental que produce.

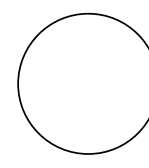

Acosta, M.V.; Z. Reicher; M. Bischoff y R.F. Turco. 1999. The role of tree leaf mulch and nitrogen fertilizer on turfgrass soil quality. Biol. Fertil. Soils 29(1), 55-61.

Arrieche, I. y O. Mora. 2005. Efecto de la aplicación de residuos orgánicos sobre el cultivo del maíz en suelos agrícolas del estado de Yaracuy, Venezuela. Bioagro $17(3), 155-159$.

Boone, F.R. y B.W. Veen. 1994. Mechanisms of crop responses to soil compaction. Developments in Agricultural Engineering 11: Soil Compaction and Crop Production. Elsevier Science, Amsterdam, The Netherlands.

\section{REFERENCIAS BIBLIOGRÁFICAS}

Bottenberg, H.; J. Masiunas y C. Eastman. 1999. Strip tillage reduces yield loss of snapbean planted in rye mulch. Hort Technol. 9(2), 235-240.

Campos, B.C.; D.J. Reinert y R. Nicolodi. 1995. Estabilidade estrutural de um Latossolo Vermelho-Escuro distrófico após sete anos de rotação de culturas e sistemas de manejo de solo. Rev. Bras. Ci. Solo 19, 121-125.

Carpenedo, V. y J. Mielniczuk. 1990. Estado de agregação e qualidade dos agregados de latossolos roxos, submetidos a diferentes sistemas de manejo. Rev. Bras. Ci. Solo 14, 99-105. 
Cuevas J.B.; S.O. Seguel; A. Ellies y J. Dörner. 2006. Efectos de las enmiendas orgánicas sobre las propiedades físicas del suelo con especial referencias a la adición de lodos urbanos. J. Soil Sci. Plant Nutr. $6(2), 1-12$

Cunha, E.; L.C. Balbino; L.F. Stone; W.M. Leandro y G.C. De Oliveira. 2007. Influência de rotações de culturas nas propriedades físico-hídricas de um Latossolo Vermelho em plantio direto. Eng. Agríc. 27(3), 665-674

Edwards, A.P. y J.M. Bremer. 1967. Microagregates in soil. J. Soil Sci. 18, 64-73.

Emerson, W.W. y D. Mcgarry. 2003. Organic carbon and soil porosity. Aust. J. Soil Res. 41, 107-118.

Fassbender, W.H. 1986. Química de suelos. IICA, San José.

Felton, G.K. 1995. Temporal variation of soil hydraulic properties on municipal solid waste amended mine soils. Trans. ASAE 38, 775-782.

Harris, R.F.; G. Chesters y O.N. Allen. 1966. Dynamics of soil aggregation. Adv. Agron. 18, 107-169.

Hernández, I.; E. Medina y H.D. López. 1995. Respiración edáfica y aportes de materia orgánica por las raíces y la hojarasca en un cultivo de caña de azúcar. Agron. Trop. 45, 12-142.

Herrick, J.E. y R. Lal. 1995. Soil physical property changes during dung cescomposition in a tropical pasture. Soil Sci. Soc. Amer. J. 59, 908-912.

Hudson, B. 1994. Soil organic matter and available water capacity. J. Soil Water Conserv. 49, 189-194.

IGAC. 2006. Métodos analíticos de laboratorio de suelos. 6a ed. Instituto Geográfico Agustín Codazzi, Bogotá.

Kay, B.D.; A.P. Da Silva y J.A. Baldock. 1997. Sensitivity of soil structure to changes in organic $C$ content: predictions using pedotransfer functions. Can. J. Soil Sci. 77, 655-667.

Khan, K.; A. Gattinger; F. Buegger; M. Schloter y R.G. Joergensen. 2008. Microbial use of organic amendments in saline soils monitored by changes in the ${ }^{13} \mathrm{C} /{ }^{12} \mathrm{C}$ ratio. Soil Biol. Biochem. 40, 1217-1224.

Le Bissonnais, Y. y D. Arrouays. 1997. Aggregate stability and assessment of soil crustability and erodability: II. Application to humic loamy soils with various organic carbon contents. Eur. J. Soil Sci. 48, $39-48$.

MacRae, A. y G.R. Mehuys. 1985. The effect green manuring on the physical properties of températe area soils. Adv. Soil Sci. 3, 71-94.

Mohee, R.R. y B. Panray. 1999. Life cycle analysis of compost incorporated sugarcane bioenergy systems in Mauritius. Biomass Bioenergy 17, 73-83.

Mulumba, L. y N.R. Lal. 2008. Mulching effects on selected soil physical properties. Soil Tillage Res. 98, 106-111.

Painuli, D.K. y L.P. Abrol. 1986. Role of some organics in improving sodic soils. Indian J. Agríe. Sel. 56(4), 267-76.

Paturau, J.M. 1969. By-products of sugar cane industry. An introduction to their industrial utilization in América. Elservier Publ. Comp., London.

Pernes-Debuyser, A. y D. Tessier. 2004. Soil physical properties affected by long-term fertilization. Eur. J. Soil Sci. 55, 505-512.

Píccolo, G.A. 1995. Efecto de diferentes cultivos utilizados como abonos verdes sobre un Rodudalf típico (Misiones, Argentina). Ciencia del Suelo 13, $101-$ 103.

Rasul, G.; A. Appuhn; T. Muller y R.G. Joergensen. 2006. Salinity induced changes in the microbial use of sugar cane fillter cake material under acidic soil conditions. Appl. Soil Ecol. 31, 1-10.

Rodriguez, G. y M. Gottret. 2006. Aprendiendo del pasado para proyectarnos hacia el futuro e impacto de la tecnología de la panela en la Hoya del Río Suárez y Cundinamarca. Corpoica; CIAT, Cali, Colombia.

Sánchez-Hernández R.; V.M. Ordaz-Chaparro; G.S. Benedicto-Valdés; C.I. Hidalgo-Moreno y D.J. Palma-López. 2006. Regeneración estructural de un suelo arcilloso por aportes de vermicompost en la chontalpa, tabasco, México. Universidad y Ciencia 22(1), 13-26.

Silva, I.R. y J. Mielniczuk. 1998. Sistemas de cultivo e características do solo afetando a estabilidade de agregados. Rev. Brasil. Ci. Solo 22(2), 311-17.

Soane, B.D.; P.S. Blackwell; J.W. Dickson y D.J. Painter. 1981. Compaction by agricultural vehicles: a review. 1. Soil and wheel characteristics. Soil Tillage Res. 1, 207-237. 
Tisdall J.M. 1994. Possible role of soil microorganisms in aggregation in soil. Plant Soil 159, 115-121.

Tisdall, J.M. y J.M. Oades. 1982. Organic matter and water-stable aggregates in soils. J. Soil Sci. 33, 141-163.

Unger, P.W. y O.R. Jones. 1998. Long-term tillage and cropping systems affect bulk density and penetration resistance of soil cropped to dryland wheat and grain sorghum. Soil Till. Res. 45, 39-57.

Van Rooyen, P. C. y H. W. Webber. 1977. Long-term effects of five ameliorantson a saline-sodiesoil of South Africa. Geoderma 19, 213-225.
Velázquez-Rodríguez, A.; D. Flores-Román y O.A. Acevedo-Sandoval. 2001. Formación de agregados en tepetate por influencia de especies vegetales. Agrociencia $35,311-320$.

Zérega, L. 1993. Manejo y uso agronómico de la cachaza en suelos cañameleros. Rev. Caña Azúcar 11(2), 71-92.

Zurro, P. 2005. Producción de abonos y fertilizantes a partir de subproductos de la industria azucarera orgánica. En: http://www.elparanaense.com.ar/pdf/ MemoriaabonosIngSanJavier MNES.pdf; consulta: noviembre de 2008. 